\title{
Condensation of MgS in outflows from carbon stars
}

\author{
S. Zhukovska and H.-P. Gail
}

\author{
Zentrum für Astronomie, Institut für Theoretische Astrophysik, Universität Heidelberg, Albert-Überle-Str. 2, 69120 Heidelberg, \\ Germany \\ e-mail: gail@ita.uni-heidelberg.de
}

Received 28 February 2008 / Accepted 4 May 2008

\begin{abstract}
Aims. The basic mechanism responsible for the widespread condensation of $\mathrm{MgS}$ in the outflows from carbon-rich stars on the tip of the AGB is discussed with the aim of developing a condensation model that can be applied in model calculations of dust formation in stellar winds.

Methods. The different possibilities for how MgS may be formed in the chemical environment of outflows from carbon stars are explored by some thermochemical calculations and by a detailed analysis of the growth kinetics of grains in stellar winds. The optical properties of core-mantle grains with an $\mathrm{MgS}$ mantle are calculated to demonstrate that such grains reproduce the structure of the observed $30 \mu \mathrm{m}$ feature. These considerations are complemented by model calculations of circumstellar dust shells around carbon stars.

Results. It is argued that MgS is formed via precipitation on silicon carbide grains. This formation mechanism explains some of the basic observed features of $\mathrm{MgS}$ condensation in dust shells around carbon stars. A weak secondary peak at about $33 \ldots 36 \mu \mathrm{m}$ is shown to exist in certain cases if $\mathrm{MgS}$ forms a coating on $\mathrm{SiC}$. This new feature seems to have occasionally been observed.
\end{abstract}

Key words. circumstellar matter - stars: mass-loss - stars: winds, outflows - stars: AGB and post-AGB

\section{Introduction}

Magnesium sulphide is a wide-spread dust component in dustforming carbon stars. Since $\mathrm{Mg}$ and $\mathrm{S}$ are abundant elements, this dust species forms a major component in the dust mixture injected by dying AGB stars to the interstellar medium. Any attempt to model the dust production by AGB stars and their contribution to the interstellar dust population requires a knowledge of the production mechanisms, at least, those of the abundant dust species. Since for $\mathrm{MgS}$ a condensation model for this species is presently lacking, we try to develop in this paper such a model. We will explain, why we believe that $\mathrm{MgS}$ condenses in stellar outflows as a mantle on $\mathrm{SiC}$ grains.

Magnesium sulphide was detected as a dust component in circumstellar dust shells enshrouding some carbon stars and in the environment of two planetary nebulae by Forrest et al. (1981) by observing a broad and prominent emission band in the farinfrared spectrum which apparently is centred around $30 \mu \mathrm{m}$. At the instant of its detection the nature of the carrier of this emission band was not immediately clear, except that the feature can only be a solid state absorption band. Goebel \& Moseley (1985) proposed $\mathrm{MgS}$ as the carrier of this feature. $\mathrm{MgS}$ has an infrared absorption feature just in the wavelength region of the newly detected feature and chemical equilibrium condensation calculations of Lattimer et al. (1978) for carbon rich element mixtures, originally performed for identifying possible supernova condensates, had shown that $\mathrm{MgS}$ should be an abundant solid phase at temperatures of the order of $600 \mathrm{~K}$ or lower. A companion paper to the paper of Goebel \& Moseley (1985) by Nuth et al. (1985) showed newly determined optical properties of a number of solid phases, $\mathrm{MgS}, \mathrm{CaS}, \mathrm{FeS}, \mathrm{SiS}_{2}, \mathrm{FeS}_{2}$, and $\mathrm{Fe}_{3} \mathrm{C}$, that could be responsible for the $30 \mu \mathrm{m}$ band and which were compared to observations in the paper of Goebel \& Moseley. Though the match between the observed feature and the absorption band of $\mathrm{MgS}$ was not perfect, the identification of $\mathrm{MgS}$ as carrier of the $30 \mu \mathrm{m}$ band has been widely accepted since that time, though other interpretations are occasionally discussed (e.g. Papoular 2000; Grishko et al. 2001; Volk et al. 2002).

In order to allow a calculation of the $\mathrm{MgS}$ emission feature in model calculations of radiative transfer in circumstellar dust shells, and thus to enable a quantitative comparison between observations and theory, Begemann et al. (1994) determined optical constants of $\mathrm{Mg}_{x} \mathrm{Fe}_{1-x} \mathrm{~S}(0.9 \geq x \geq 0)$. They obtained good agreement between the observed emission band profile and the calculated spectrum in the $30 \mu \mathrm{m}$ region for a radiative transfer model of the circumstellar dust shell of IRC+10216, which left little doubt with respect to the interpretation of the $30 \mu \mathrm{m}$ emission band as being due to $\mathrm{MgS}$.

A number of observational and theoretical studies of the $30 \mu \mathrm{m}$ emission from dust enshrouded AGB and post-AGB stars, and from the environment of planetary nebulae have appeared since that time (e.g. Omont 1993; Omont et al. 1995; Yamamura et al. 1998; Jiang et al. 1999; Szczerba et al. 1999; Hrivnak et al. 2000; Hony et al. 2002; Volk et al. 2002; Hony \& Bouwmann 2004; Lagadek et al. 2006; Zijlstra et al. 2006; Leisenring et al. 2008). Good examples of the emission band can be found in Hony et al. (2002), Volk et al. (2002) for galactic objects and Lagadek et al. (2006) and Zijlstra et al. (2006) for the Magellanic clouds. They all show unequivocally that $\mathrm{MgS}$ formation is a common phenomenon for carbon stars on the tip of the AGB.

The formation mechanism of this $\mathrm{MgS}$ is not clear. It was already speculated in the first papers by Nuth et al. (1985) and Goebel et al. (1985) that MgS forms via a surface reaction on carbon grains and this scenario was favoured over MgS condensation as a separate dust species. A laboratory investigation on MgS condensation was conducted by Kimura et al. (2005), but 


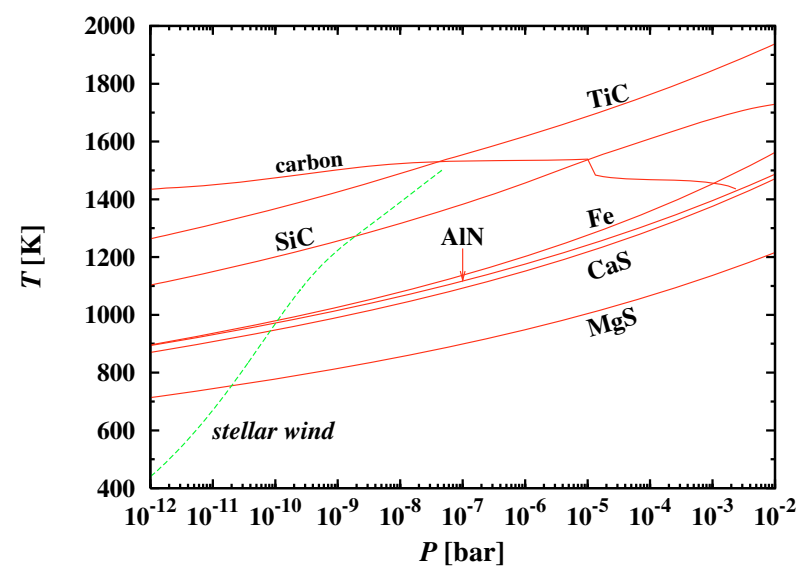

Fig. 1. Stability limits of solid phases formed from the most abundant elements in chemical equilibrium for a carbon rich element mixture $\left(\epsilon_{\mathrm{C}}=1.2 \epsilon_{\mathrm{O}}\right)$. The dashed line shows the $P-T$-trajectory of a stationary dust driven stellar wind with a mass-loss rate of $\dot{M}=10^{-5} M_{\odot} \mathrm{yr}^{-1}$

this gives no direct insight into the formation process. No further discussion of this problem seems to have appeared in the astrophysical literature so far. In this paper we will show that $\mathrm{MgS}$ can only be formed in the outflows of stars by precipitating on pre-existing grains and that $\mathrm{MgS}$ in all likelyhood forms as mantle on the silicon carbide grains formed closer to the star at higher temperatures.

The plan of the paper is as follows: first, we discuss some aspects of the chemistry associated with sulphur bearing condensed phases in Sect. 2. The kinetics of dust particle growth in a stellar outflow is discussed in Sect. 3. The results of some model calculations for $\mathrm{MgS}$ condensation in stellar winds are presented in Sect. 4 and the optical properties of grains formed by $\mathrm{MgS}$ grown on a silicon carbide or carbon core are briefly discussed in Sect. 5. Our final conclusions are presented in Sect. 6.

\section{Stability of sulphur compounds}

We start with a brief discussion of the equilibrium chemistry of sulphur compounds in a carbon rich element mixture. Figure 1 shows the results of a calculation of the chemical equilibrium composition of an element mixture with cosmic element abundances (e.g. Grevesse \& Sauval ${ }^{1}$ 1998) with carbon abundance set to 1.2 times the oxygen abundance. The lines in this $P-T$ diagram correspond to the upper stability limits of the indicated solid phases. For comparison we also show the wind trajectory of a stationary dust-driven wind model for the outflow from a carbon star. In unconditional chemical equilibrium also some oxygen bearing condensed phases would exist at temperatures below $800 \mathrm{~K}$ (cf. Lattimer et al. 1978). The oxygen for their formation has to come from a reduction of $\mathrm{CO}$. It is unlikely that such reactions are kinetically possible and we assumed here that the oxygen is completely blocked in $\mathrm{CO}$ which does not react with other species in the system, i.e., we consider a conditional chemical equilibrium where $\mathrm{O}$ is arrested in $\mathrm{CO}^{2}$.

From the abundant elements with abundances relative to $\mathrm{H}$ exceeding $10^{-6}$, only $\mathrm{CaS}$ and $\mathrm{MgS}$ are formed as solid sulphur

\footnotetext{
${ }_{1}$ We prefer these over the more recent data of Asplund et al. (2005) since the reduction of the $\mathrm{C}$ and $\mathrm{O}$ abundances in the new table are in clear contradiction to the results of helioseismology (e.g. Chaplin et al. 2007).

2 Some condensation temperatures then are somewhat different from the results of more conventional condensation calculations.
}

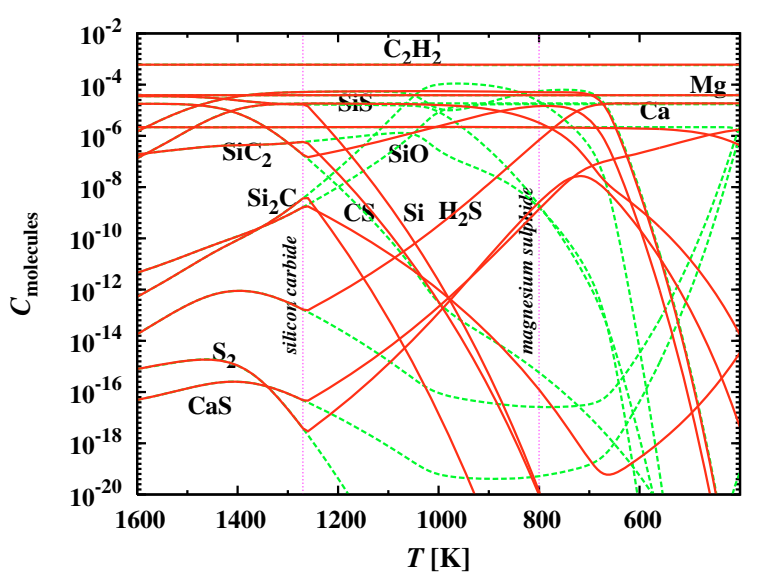

Fig. 2. Concentrations in the gas phase of $\mathrm{S}, \mathrm{Si}$, and $\mathrm{Mg}$ atoms and their abundant molecular compounds with $\mathrm{H}, \mathrm{C}, \mathrm{O}$, and $\mathrm{S}$ for a cooling sequence with $P=10^{-10}$ bar if formation of solid $\mathrm{SiC}$ in chemical equilibrium is allowed for (full lines) and if the formation of $\mathrm{SiC}$ is suppressed (dashed lines)

bearing phases in chemical equilibrium. Troilite (FeS), that is abundant in oxygen rich environments, is not formed in the carbon rich environment because $\mathrm{MgS}$ is more stable than $\mathrm{FeS}$, and in a carbon rich environment the $\mathrm{Mg}$ is not consumed by the even more stable magnesium silicates as in the oxygen rich case. The first sulphur bearing solid compound that appears in a cooling sequence at pressure $10^{-10}$ bar is $\mathrm{CaS}$ at about $950 \mathrm{~K}$, followed by $\mathrm{MgS}$ at about $750 \mathrm{~K}$. The temperatures where $\mathrm{CaS}$ and $\mathrm{MgS}$ become stable in a stellar outflow depend somewhat on the mass-loss rate and are of the just mentioned order of magnitude for a mass-loss rate of $\dot{M}=10^{-5} M_{\odot} \mathrm{yr}^{-1}$ or somewhat less for smaller values of $\dot{M}$.

Calcium sulphide may be formed in a carbon rich environment prior to MgS condensation, but because of the low calcium abundance (which is only $1 / 17$ of the $\mathrm{Mg}$ abundance) it would be difficult to detect this dust component in infrared spectra from circumstellar dust shells in the presence of $\mathrm{MgS}$ dust. The formation of $\mathrm{CaS}$ cannot consume all of the $\mathrm{S}$ since the sulphur abundance is about eight times the $\mathrm{Ca}$ abundance, i.e., $\mathrm{MgS}$ is formed in addition to $\mathrm{CaS}$ at lower temperatures. Since the Mg abundance is about twice that of the $\mathrm{S}$ abundance, at most half of the available $\mathrm{Mg}$ can be consumed by the formation of $\mathrm{MgS}$. The remaining fraction of $\mathrm{Mg}$ stays in the gas phase and may or may not precipitate later on other condensed phases.

The abundance of $\mathrm{S}$ bearing molecules in the gas phase is shown in Fig. 2 for a cooling sequence with total pressure $10^{-10}$ bar that is representative for the pressure in the dust formation zone of a stellar wind (cf. Fig. 1). The figure shows the composition of the gas phase in a restricted chemical equilibrium where carbon is not completely condensed into solid carbon, a situation encountered in an outflow, where carbon formation cannot keep pace with cooling and only part of the carbon is converted from gaseous species into the condensed phase. Results for the sulphur chemistry are shown for the two cases that (i) the $\mathrm{Si}$ condenses into silicon carbide $(\mathrm{SiC})$ at temperatures below about $1250 \mathrm{~K}$; and (ii) for the case that formation of $\mathrm{SiC}$ is suppressed. A comparison of the results for both cases shows:

- If no $\mathrm{SiC}$ is formed, the sulphur is almost completely bound in SiS below $1500 \mathrm{~K}$ and no other S bearing species are abundant in the gas phase.

- Only if the $\mathrm{Si}$ is consumed by the formation of solid $\mathrm{SiC}$, is the sulphur liberated to form increasing amounts of other 
S-bearing molecules - in particular $\mathrm{H}_{2} \mathrm{~S}$ - in pace with conversion of $\mathrm{Si}$ from $\mathrm{SiS}$ into silicon carbide.

This means that the sulphur chemistry of the gas phase and the formation of S-baring solid phases are strongly coupled to the process of silicon carbide condensation.

As long as not much solid silicon carbide has formed the sulphur is blocked in the rather stable SiS molecules and the formation of $\mathrm{MgS}$ from abundant gas phase species requires the following reaction

$\mathrm{SiS}+\mathrm{Mg} \stackrel{\mathrm{k}_{1}}{\longrightarrow} \mathrm{MgS}(\mathrm{s})+\mathrm{Si}$

The change in free enthalpy by this reaction is $\Delta G_{1}=$ $-30 \mathrm{~kJ} / \mathrm{mol}$ at $800 \mathrm{~K}$ (thermochemical data are from Chase 1998). After the consumption of SiS by silicon carbide formation and formation of abundant $\mathrm{H}_{2} \mathrm{~S}$ the following reaction becomes possible

$\mathrm{H}_{2} \mathrm{~S}+\mathrm{Mg} \stackrel{\mathrm{k}_{2}}{\longrightarrow} \mathrm{MgS}(\mathrm{s})+\mathrm{H}_{2}$.

The change in free enthalpy in this case is $\Delta G_{2}=-350 \mathrm{~kJ} / \mathrm{mol}$. The corresponding laws of mass action for these two chemical reactions are

$a_{\mathrm{MgS}(\mathrm{s})} p_{\mathrm{Si}}=p_{\mathrm{SiS}} p_{\mathrm{Mg}} \mathrm{e}^{-\Delta G_{1} / R T}$

$a_{\mathrm{MgS}(\mathrm{s})} p_{\mathrm{H}_{2}}=p_{\mathrm{H}_{2} \mathrm{~S}} p_{\mathrm{Mg}} \mathrm{e}^{-\Delta G_{2} / R T}$

where $a_{\mathrm{MgS}(\mathrm{s})}$ is the activity of solid $\mathrm{MgS}$. In chemical equilibrium the activity of the solid compound equals unity. These equations determine the equlibrium pressure in Sect. 3.2.

\section{Formation of MgS in a stellar wind}

In stellar outflows temperature and density variations occur on shorter timescales than particle growth. In this case condensation proceeds far from chemical equilibrium and particle growth has to be treated by reaction kinetics. We consider here the conditions under which $\mathrm{MgS}$ condensation may occur in a rapidly expanding stellar outflow.

\subsection{The wind model}

\subsubsection{Stationary wind model}

The formation of $\mathrm{MgS}$ in stellar outflows from AGB stars seems to be associated with late phases of AGB evolution of low and intermediate mass stars, where the mass-loss rate is very high and the stars have already lost most of their hydrogen rich envelope.

At the tip of the AGB, radiation pressure on dust becomes the dominating driving source of the wind. The underlying stars all seem to be variables, either Miras or LPVs. The structure of the outflow is complicated in this case, since there are always several shocks running outwards, which are superposed on the average outflow of stellar material. This is demonstrated by several model calculations of dust forming stellar outflows of pulsating stars (e.g. Bowen 1988; Fleischer et al. 1991, 1992; Feuchtinger et al. 1993; Höfner \& Dorfi 1997; Höfner et al. 1998; Winters et al. 1997, 2000; Jeong et al. 2003).

Calculating dust formation rates for multi-component dust mixtures of stars with quite different elemental compositions and widely varying stellar parameters presently seems impossible for reasons of computational time requirements if the shock structure of the wind is to be taken into account. For the purpose of calculating the composition of the dust mixture and the amount of dust formed in the outflows of AGB-stars we use a simpler model, which assumes a stationary outflow. If one compares the velocity and density profiles of such stationary winds with published models of dust forming pulsators (e.g. Winters et al. 2000; Jeong et al. 2003), one observes in most cases a strong resemblance of the average velocity and density profiles with that of the stationary models, except that in the pulsation models outward propagating shocks are superposed on the average flow structure. So we can hope to obtain at least an estimate of the average quantities of dust formed in the outflow if dust formation is calculated for such an average outflow structure. One important consequence of this neglect of the detailed structure of the flow is that we cannot determine self-consistently the mass-loss rate $\dot{M}$. We have to treat this as a free parameter, which has to be fixed in some other way.

\subsubsection{Stellar parameters}

The central star is assumed to be on the tip of the AGB. Its luminosity then is typically $L_{*}=1.5 \times 10^{4} L_{\odot}$.

For stars forming $\mathrm{MgS}$ in their outflow a rather high metallicity is required since at low metallicities the abundances of $\mathrm{Mg}$ and $\mathrm{S}$ would be too low for this to be possible. Only a small fraction of the carbon stars in the Small Magellanic Cloud, for instance, seem to form $\mathrm{MgS}$ dust (Sloan et al. 2006), while for carbon stars in the Large Magellanic Cloud the formation of $\mathrm{MgS}$ seems to be more common (Zijlstra et al. 2006), and in the Milky Way it is quite common for highly evolved carbon stars (e.g. Hony 2002). We note already that according to observations $\mathrm{MgS}$ formation seems to be associated with $\mathrm{SiC}$ formation: Stars showing the $\mathrm{MgS}$ feature also show the $\mathrm{SiC}$ feature, but not all stars showing a $\mathrm{SiC}$ feature also show the $\mathrm{MgS}$ feature in their spectra (e.g. Zijlstra 2006). In order that low and intermediate mass stars of Pop I (or not much lower) metallicity become carbon stars, their initial masses must be from the mass range between about $1.5 M_{\odot}$ and about $4 M_{\odot}$.

After massive mass-loss on the AGB the stellar mass is likely to be substantially reduced and we assume in the model calculation a fixed stellar mass of $1 M_{\odot}$. The stellar radiation is approximated by a black body spectrum. The effective temperature (in the inter-pulse phase) is determined from the relation

$$
\log T_{\text {eff }}=.234 \log M-.2 \log L-.116 \log \frac{Z}{0.02}+4.146
$$

given by Vassiliadis \& Wood (1993) for stars on the AGB. The mass $M$ and luminosity $L$ are in solar units. With the assumed stellar parameters and solar metallicity one finds $T_{\text {eff }}=2250 \mathrm{~K}$.

The mass-loss rate is estimated from the relation

$\dot{M}=2.1 \times 10^{-8} \frac{L}{v_{\infty}}$

given by Vassiliadis \& Wood (1993) for stars in the superwind phase. The luminosity is in units of $L_{\odot}$, the outflow velocity $v_{\infty}$ in $\mathrm{km} \mathrm{s}^{-1}$, and the mass-loss rate in units of $M_{\odot} \mathrm{yr}^{-1}$. With the estimated stellar parameters and a typical outflow velocity in the superwind phase of $10 \ldots 15 \mathrm{~km} \mathrm{~s}^{-1}$ one obtains a mass-loss rate of about $\dot{M}=2 \ldots 3 \times 10^{-5} M_{\odot} \mathrm{yr}^{-1}$ for stars at the end of their AGB evolution. A value of $\dot{M}=3 \times 10^{-5} M_{\odot} \mathrm{yr}^{-1}$ is used in the model calculations.

A typical run of density and temperature in a stationary stellar wind model of a carbon star calculated for a dust driven stationary wind model (cf. Gail \& Sedlmayr 1986, 1987 for the details of how such models are constructed) is shown in Fig. 1. 
The $\mathrm{C} / \mathrm{O}$ abundance ratio of the outflowing material in any case exceeds unity since $\mathrm{MgS}$ is not formed in an oxygen rich environment.

\subsection{Dust particle growth}

The model calculation of condensation in the outflow from a carbon star considers the following dust components: Carbon dust, silicon carbide dust, and iron dust. The formation of these dust species is calculated as outlined in Ferrarotti \& Gail (2006). Here we add the growth of magnesium sulphide to our model. The dust formation is calculated by solving the equations for dust growth (e.g. Gail 2003)

$\frac{\mathrm{d} a}{\mathrm{~d} t}=V_{0} \alpha n_{\mathrm{gr}} \sqrt{\frac{k T_{\mathrm{g}}}{2 \pi m_{\mathrm{gr}}}}\left\{\Phi(w)-\frac{p_{\mathrm{eq}}\left(T_{\mathrm{d}}\right)}{n_{\mathrm{gr}} k T_{\mathrm{d}}} \sqrt{\frac{T_{\mathrm{d}}}{T_{\mathrm{g}}}}\right\}$

for all species of interest. Here $a$ is the radius of a dust grain, $V_{0}$ the volume of one formula unit ${ }^{3}$ of the chemical compound in the solid, $\alpha$ is the growth coefficient, $n_{\mathrm{gr}}$ is the particle density of the growth species in the gas phase, $m_{\mathrm{gr}}$ its mass, $p_{\mathrm{eq}}$ is the partial pressure of the growth species in a state of chemical equilibrium between the condensed phase and the gas phase, and $T_{\mathrm{g}}$ and $T_{\mathrm{d}}$ are the gas temperature and the internal lattice temperature of the dust, respectively. The quantity

$\Phi=\sqrt{1+\frac{\pi \mu m_{\mathrm{H}}}{8 k T_{\mathrm{g}}} w^{2}}$

depends in principle on the drift velocity $w$ of the grains relative to the gas. Here drift is neglected and we put $\Phi=1$ since for high mass-loss rates drift velocities are small compared to the sonic velocity. The volume $V_{0}$ of one chemical formula unit occupied in the solid is

$V_{0}=\frac{A_{\mathrm{d}} m_{\mathrm{H}}}{\varrho_{\mathrm{d}}}$,

where $A_{\mathrm{d}}$ is the atomic weight of the chemical formula unit and $\varrho_{\mathrm{d}}$ the bulk density of the condensate.

The growth equation has to be complemented by an equation for the consumption of the growth species from the gas phase. The details are described in the paper by Ferrarotti \& Gail (2006) and are not repeated here.

As the rate determining step for the formation of $\mathrm{MgS}$ via reaction (2) we assume the addition of a $\mathrm{H}_{2} \mathrm{~S}$ molecule from the gas phase since this is the least abundant of the molecules involved in the reaction. $p_{\text {eq }}$ in Eq. (7) is calculated from Eq. (4) with activity $a_{\mathrm{MgS}}=1$. The thermodynamic data for calculating $\Delta G$ are taken from the JANAF-tables (Chase 1998).

The growth coefficient $\alpha$, the probability that a collision is followed by a growth step, seems not to be known. For the similar solid $\mathrm{MgO}$ with the same structure and similar bonding properties Hashimoto (1990) measured a growth coefficient of about 0.2 and we will use the same value for $\mathrm{MgS}$. Results for growth coefficients of $\mathrm{Zn}$ obtained under microgravity conditions seem to be much smaller (e.g. Michael et al. 2003), but it is unclear whether this can be applied to the present case.

\subsection{Conditions for grain growth}

Some insight into the origin of the difficulty to explain how $\mathrm{MgS}$ forms in the outflow can be gained from considering a simplified

\footnotetext{
3 The formula unit corresponds to the chemical formula of the condensed phase, if it is written with integer stoichiometric coefficients.
}

growth model. We neglect in the growth equation (i) the vaporization and (ii) the consumption of the growth species by grain growth. Additionally we use in the expression for the thermal velocity a representative value for the temperature in the growth zone of the dust. The first factor is generally not too bad for dust growth problems since the vapour pressure $p_{\text {eq }}$ usually decreases rapidly with decreasing temperature below the stability limit of a solid. The second factor restricts the applicability of our considerations to the early growth phase before the gas phase becomes exhausted of condensable material. This initial growth phase is that which we now consider.

The particle density $n_{\mathrm{gr}}$ is the least abundant gas phase species involved in the rate determining reaction for the growth. Its abundance is usually some fraction $g$ of the least abundant element from which this gas phase species is composed. In our case, where reaction (2) is assumed to be the key reaction for $\mathrm{MgS}$ growth, the least abundant growth species is $\mathrm{H}_{2} \mathrm{~S}$ and sulphur is the least abundant of the elements forming this molecule. We write $n_{\mathrm{gr}}=g \epsilon_{\mathrm{el}} N_{\mathrm{H}}$, where $N_{\mathrm{H}}$ is the number density of $\mathrm{H} \mathrm{nu}-$ clei which can be calculated from the mass density $\rho$ of the wind

$$
N_{\mathrm{H}}=\frac{\rho}{\left(1+4 \epsilon_{\mathrm{He}}\right) m_{\mathrm{H}}} .
$$

The mass density is given in a stationary outflow with mass-loss rate $\dot{M}$ and outflow velocity $v$ by

$\rho=\frac{\dot{M}}{4 \pi r^{2} v}$.

Since the condensation of $\mathrm{MgS}$ becomes possible only at a temperature much lower than the condensation temperature of carbon dust, the radiation pressure on carbon dust has driven the outflow velocity already nearly to the terminal outflow velocity of the stellar wind if $\mathrm{MgS}$ condensation commences. For the problem of $\mathrm{MgS}$ condensation we can consider the wind velocity $v$ therefore to be constant. By changing the independent variable $t$ to $r$ by means of $v \mathrm{~d} t=\mathrm{d} r$ we can write the equation for the initial growth of dust as

$\frac{\mathrm{d} a}{\mathrm{~d} r}=V_{0} \alpha v_{\mathrm{gr}} \frac{\dot{M}}{1.4 m_{\mathrm{H}} 4 \pi r^{2} v^{2}}$.

Here $v_{\mathrm{gr}}$ is the thermal velocity of the growth species (assumed to be constant). Integrating from the inner radius $R_{\mathrm{d}}$ of the onset of growth of the dust species to infinity we obtain

$a_{\infty}=a_{0}+V_{0} \alpha v_{\mathrm{gr}} g \epsilon_{\mathrm{el}} \frac{\dot{M}}{1.4 m_{\mathrm{H}} 4 \pi R_{\mathrm{d}} v^{2}}$,

where $a_{0}$ is the radius of the seed nuclei for dust growth and $a_{\infty}$ the radius at infinity. This equation requires that the grain radius $a_{\infty}$ remains smaller than the maximum radius $a_{\max }$ attained if all condensable material is condensed. This maximum possible radius is given by

$\frac{4 \pi}{3} a_{\max }^{3} \epsilon_{\mathrm{d}}=\frac{V_{0} \epsilon_{\mathrm{key}}}{v_{\mathrm{key}}}$

Here $\epsilon_{\mathrm{d}}$ is the number of dust grains per hydrogen nucleus, $\epsilon_{\mathrm{key}}$ the key element for the formation of the dust species considered, and $v_{\text {key }}$ the number of atoms of the key element in the chemical formula of the solid.

We can now write

$a_{\infty}=a_{0}+a_{\max } \frac{\dot{M}}{\dot{M}_{\mathrm{cr}}}$ 
Table 1. Basic data used for calculation of $\mathrm{MgS}$ grain growth.

\begin{tabular}{lrl}
\hline \hline Quantity & Value & Unit \\
\hline$A$ & 56.37 & \\
$\varrho_{\mathrm{d}}$ & 2.68 & $\mathrm{~g} \mathrm{~cm}^{-3}$ \\
$V_{0}$ & $3.51 \times 10^{-23}$ & $\mathrm{~cm}^{3}$ \\
$\alpha$ & 0.2 & \\
$\epsilon_{\mathrm{Mg}}$ & $3.85 \times 10^{-5}$ & \\
$\epsilon_{\mathrm{Si}}$ & $3.58 \times 10^{-5}$ & \\
$\epsilon_{\mathrm{S}}$ & $1.85 \times 10^{-5}$ & \\
$\epsilon_{\mathrm{d}}$ & $10^{-13}$ & \\
$a_{\max }$ & 0.12 & $\mu \mathrm{m}^{-1}$ \\
$v_{\text {th }}$ & $1.52 \times 10^{4}$ & $\mathrm{~cm} \mathrm{~s}^{-1}$ \\
\hline
\end{tabular}

with

$\dot{M}_{\mathrm{cr}}=\frac{a_{\mathrm{max}} 1.4 m_{\mathrm{H}} 4 \pi R_{\mathrm{d}} v^{2}}{V_{0} \alpha v_{\mathrm{gr}} g \epsilon_{\mathrm{el}}}$.

The quantity $\dot{M}_{\text {cr }}$ defines a critical mass-loss rate for particle growth. If the mass-loss rate $\dot{M}$ of the stellar outflow is lower than $\dot{M}_{\text {cr }}$, the final particle radius of dust grains of the considered kind in the outflow is smaller than the maximum radius attained in case of complete condensation. Condensation by growth starting with some kind of small seed particles is always incomplete in this case and most of the condensable material remains in the gas phase. If, however, the mass-loss rate $\dot{M}$ of the stellar outflow is of the order of $\dot{M}_{\text {cr }}$ or exceeds $\dot{M}_{\text {cr }}$, the final particle radius in the outflow approaches $a_{\max }$; complete condensation is possible in this case by growth starting with some kind of small seed particles (at least this is not kinetically forbidden).

Applying this to $\mathrm{MgS}$ condensation we obtain

$\dot{M}_{\mathrm{cr}, \mathrm{MgS}}=5.4 \times 10^{-4} M_{\odot} \mathrm{yr}^{-1}$.

Here we assumed a typical outflow velocity of $v=10 \mathrm{~km} \mathrm{~s}^{-1}$ and a radius of $R_{\mathrm{d}}=2 \times 10^{14} \mathrm{~cm}$ corresponding to about 5 stellar radii. As the key element for $\mathrm{MgS}$ condensation we assume sulphur since it has lower abundance than $\mathrm{Mg}$. The growth species is $\mathrm{H}_{2} \mathrm{~S}$. As a typical temperature for calculating $v_{\mathrm{gr}}$ we choose $600 \mathrm{~K}$. All other quantities are given in Table 1. The critical mass-loss rate is about a factor of ten higher than the highest mass-loss rates observed for highly evolved AGB stars (e.g. van Loon et al. 1999). As a result the radius can approach at most $1 / 10$ of that for complete condensation and only about $1 / 1000$ of the condensable material can be condensed into $\mathrm{MgS}$ grains if the dust grains start their growth for instance from seed particles with sizes of the order of $1 \mathrm{~nm}$ that are typical for seed particles resulting from nucleation from the gas phase.

For such a low fraction of condensation of $\mathrm{S}$ and $\mathrm{Mg}$ into $\mathrm{MgS}$ this dust component would not be detectable in the infrared emission from circumstellar dust shells of AGB stars. Since MgS is frequently observed in highly evolved AGB stars, it cannot be formed by condensation from the gas phase via nucleation and subsequent growth. We therefore need to look for some other formation mechanism.

\subsection{Heterogeneous growth}

The most promising alternative is heterogeneous growth on some other kind of dust particles that already have moderate radii at the onset of $\mathrm{MgS}$ growth in order that the frequency of collisions of such a particle with growth species from the gas phase is high from the beginning. We derive the condition for this type of growth to occur by considering the initial growth phase, where the growth of a coating of $\mathrm{MgS}$ on some type of carrier grain has not yet significantly increased the total grain radius.

We use essentially the same type of simplifications as before and consider the following equation for the increase of the volume $V$ of the coating on the surface of carrier grains of radius $a_{\mathrm{c}}$

$\frac{\mathrm{d} V}{\mathrm{~d} t}=V_{0} \alpha v_{\mathrm{gr}} n_{\mathrm{gr}} 4 \pi a_{\mathrm{c}}^{2}$.

Introducing again $r$ as the independent variable we obtain upon integrating this equation from the radius $R_{\mathrm{d}}$ of the onset of formation of the coating to infinity

$V_{\infty}=V_{0} \alpha v_{\mathrm{gr}} 4 \pi a_{\mathrm{c}}^{2} g \epsilon_{\mathrm{el}} \frac{\dot{M}}{1.4 m_{\mathrm{H}} 4 \pi R_{\mathrm{d}} v^{2}}$.

This is the final volume of the coating at large distances. The maximum possible volume $V_{\max }$ of the coating on a carrier dust grain is, again, given by Eq. (14). We can now write

$V_{\infty}=V_{\max } \frac{\dot{M}}{\dot{M}_{\text {het }}}$,

where

$\dot{M}_{\mathrm{het}}=\frac{\epsilon_{\mathrm{key}}}{v_{\mathrm{key}} \epsilon_{\mathrm{d}}} \frac{1.4 m_{\mathrm{H}} R_{\mathrm{d}} v^{2}}{\alpha v_{\mathrm{gr}} a_{\mathrm{c}}^{2} g \epsilon_{\mathrm{el}}}$

is a characteristic mass-loss rate of the stellar outflow for formation of coatings on the surface of pre-existing grains. If the mass-loss rate $\dot{M}$ of the stellar outflow is less than the characteristic mass-loss rate $\dot{M}_{\text {het }}$ only part of the condensable material will condense in the form of a coating on the surface on some carrier grains. If the mass-loss rate $\dot{M}$ of the stellar outflow exceeds the characteristic mass-loss rate $\dot{M}_{\text {het }}$ the volume of the coating approaches the maximum possible volume and complete condensation is possible, at least in principle.

Inserting numerical values for $\mathrm{MgS}$ condensation and assuming that the carrier grains for the formation of the coating have a radius of $a_{\mathrm{c}}=0.1 \mu \mathrm{m}$ one obtains

$\dot{M}_{\text {het }}=2.8 \times 10^{-4}\left(\frac{0.1 \mu \mathrm{m}}{a_{\mathrm{c}}}\right)^{2} M_{\odot} \mathrm{yr}^{-1}$.

This characteristic mass-loss rate, again, is about a factor of ten higher than the highest mass-loss rates observed for AGB stars, but since it now refers to the volume we have the result that at the end of the AGB evolution up to about $10 \%$ of the condensable material for $\mathrm{MgS}$ formation can really be condensed as a coating on suitable carrier grains, if such exist. If the core grains have a larger radius than the assumed $0.1 \mu \mathrm{m}$, or if the mass-loss rate should be exceptionally high then the fraction of condensed material may be even larger. Such amounts of condensed MgS should be clearly visible in the infrared spectrum.

Hence, we come to the conclusion that the observed $\mathrm{MgS}$ in the outflows from carbon stars on the AGB does not form as a separate dust species but condenses as a coating on the surface on some different kind of carrier grains. The formation of significant amounts of $\mathrm{MgS}$ dust requires mass-loss rates of at least a few times $10^{-5} M_{\odot} \mathrm{yr}^{-1}$, i.e., this is possible only during the superwind phase short before termination of AGB evolution if the mass-loss rates have grown to very high values. This is just what one seems to observe.

As a side-remark we note the following: Since the available amount of $\mathrm{S}$ does not suffice for the formation of thick coatings 
of $\mathrm{MgS}$ such that the final volume is much bigger than the volume of the carrier grains, the volume of condensed material on the surface of the carrier grains increases quadratically with the radius of the carrier grains (cf. Eq. (19)). Therefore it may well be that most of the $\mathrm{MgS}$ is concentrated on a sub-population of larger-than-average grains, if one has a broad distribution of grain sizes for the possible carrier grains.

\subsection{Possible carrier grains}

The dust species that may serve as carrier grains for $\mathrm{MgS}$ formation need to form dust grains with a size not much smaller than about $a_{\mathrm{c}}=0.1 \mu \mathrm{m}$ because otherwise the condensed fraction of $\mathrm{MgS}$ becomes too small if the radius of the cores is smaller than this. This restricts the possibilities as to which dust species may serve as substrates of $\mathrm{MgS}$ growth, to the most abundant dust species.

The outflowing gas enters the temperature regime below about $650 \mathrm{~K}$ favourable for $\mathrm{MgS}$ condensation with two major dust species that have grown farther inside in the shell to the required size: $\mathrm{SiC}$ grains and carbon grains. From investigations of presolar dust grains it is known that from the many polytypes of $\mathrm{SiC}$ only two are formed in circumstellar environments (Daulton et al. 2003): the cubic 3C poly-type (usually denoted as $\beta$-SiC) is found in $79.4 \%$ of all observed grains, and the hexagonal $2 \mathrm{H}$ poly-type (usually denoted as $\alpha$-SiC) is found in $2.7 \%$ of all observed grains. In $17.1 \%$ of all grains the material is an intergrowth of these two poly-types, the remaining fraction of $0.8 \%$ being other polytypes or disordered.

The lattice structure of the $\mathrm{SiC}$ grains in stellar outflows, thus, in most cases is cubic. Magnesium sulphide also has a cubic lattice structure. The electronegativity difference (cf. Pauling 1960) of $\mathrm{MgS}$ is 1.3 and that of $\mathrm{SiC}$ is 0.7 . Both compounds therefore show a significant ionic contribution to their bonding. There are obvious similarities of structure and bonding properties of $\mathrm{SiC}$ and $\mathrm{MgS}$. On the other hand, no such similarity exists between $\mathrm{MgS}$ and solid carbon. This suggests that $\mathrm{SiC}$ is much better suited as a substrate for $\mathrm{MgS}$ precipitation than carbon. We therefore propose that $\mathrm{MgS}$ grows on $\mathrm{SiC}$ grains.

A possible core grain is any compound that has a lattice structure similar to the $\mathrm{MgS}$ lattice structure and a higher condensation temperature. Among the compounds possibly formed in the stellar outflows $\mathrm{CaS}$ also satisfies both conditions. It has a crystal structure, that is the closest to that of $\mathrm{MgS}$ and becomes stable at a more than $200 \mathrm{~K}$ higher temperature than $\mathrm{MgS}$ (Fig. 1). The abundance of $\mathrm{Ca}$ in a stellar wind, however, is 16 times lower than the $\mathrm{Mg}$ abundance, which is not very favourable for its potential role as the core grain for $\mathrm{MgS}$ growth. Nevertheless, we will also check this possibility.

\section{Models with core-mantle grains}

We performed model calculations of dust formation in stellar outflows according to the wind model described in Sect. 3 to check the assumption of the core-mantle growth scenario of $\mathrm{MgS}$ formation. Grain growth is calculated as described in Sect. 3.2.

First we check the model of MgS condensation on tiny seed nuclei. The existence of such seeds of $1 \mathrm{~nm}$ is taken for granted and the growth of $\mathrm{MgS}$ is calculated. This results in grain sizes of only about $0.02 \mu \mathrm{m}$ and a very low fraction of $\mathrm{Mg}$ bound in dust, the condensation degree, of about 0.01 . This is in line with our analytical findings in Sect. 3.3. The evolution of grain radius and condensation degree for this kind of model are shown

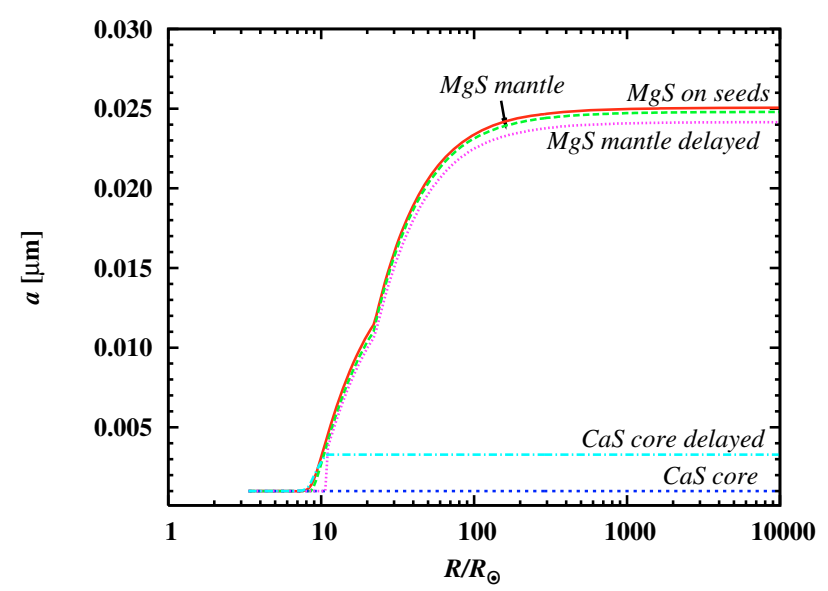

Fig. 3. Evolution of $\mathrm{MgS}$ and $\mathrm{CaS}$ grain radius as function of distance from the centre of the star, for $\mathrm{MgS}$ formation (i) on seed nuclei of $1 \mathrm{~nm}$ size; and (ii) as mantle on a CaS core. Also shown are the results of a model with delayed onset of $\mathrm{MgS}$ condensation on $\mathrm{CaS}$ core; details are given in the text.

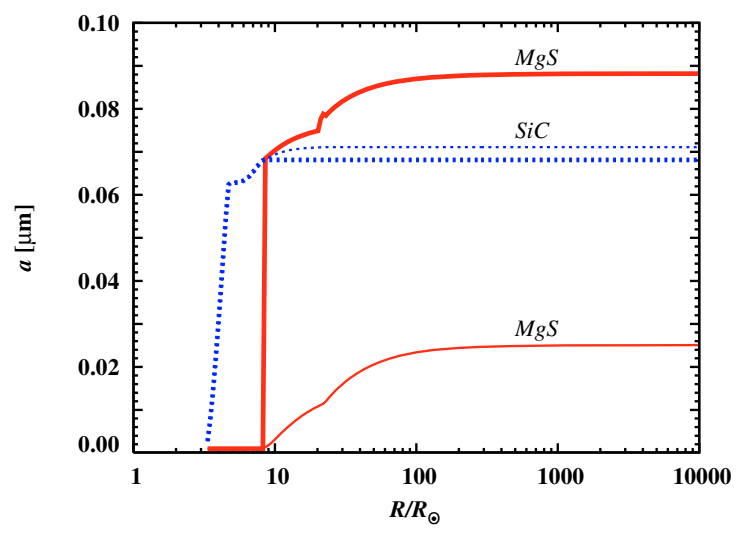

Fig. 4. Evolution of radii of $\mathrm{SiC}$ and $\mathrm{MgS}$ grains as predicted (i) by the model for MgS condensation on seed nuclei; and (ii) as a mantle on a $\mathrm{SiC}$ core (thin and thick lines, respectively).

in Figs. 3 and 5, respectively. The small amount of condensed $\mathrm{MgS}$ found in this case is obviously not enough to explain the observed $30 \mu \mathrm{m}$ emission in the spectra of carbon stars, underlining, again, the need for a core-mantle growth mechanism for $\mathrm{MgS}$ formation.

Next we check an alternative growth scenario for $\mathrm{MgS}$ formation, in which first $\mathrm{CaS}$ condenses on seed nuclei by the analogue of reaction (2), and then $\mathrm{MgS}$ grows as a mantle on this core. The model calculations show that the size of CaS grains is much smaller than the required radius of $0.1 \mu \mathrm{m}$. We also tested the case that $\mathrm{MgS}$ condensation is delayed by imposing the additional condition for the growth rate $J_{\mathrm{gr}, \mathrm{MgS}} \geq 3 J_{\mathrm{gr}, \mathrm{CaS}}$ to gain additional time for $\mathrm{CaS}$ core growth in order to get bigger grains. This artificial delay results, indeed, in bigger CaS cores, but this does not help to increase the final size of the $\mathrm{MgS}$ mantle. The results of these calculations of the evolution of the $\mathrm{MgS}$ grain radius with distance from the centre of a star for normal and delayed condensation on $\mathrm{CaS}$ are also shown in Fig. 3. The figure shows that the condensation of $\mathrm{MgS}$ on a CaS core results in almost the same grain radius as the growth of $\mathrm{MgS}$ on seeds grains. Neither of these formation mechanisms can provide the amount of $\mathrm{MgS}$ dust that is observed to condense in stellar outflows.

$\mathrm{SiC}$ grain growth, however, commences at higher temperature and proceeds faster than $\mathrm{CaS}$ growth, so that at the 


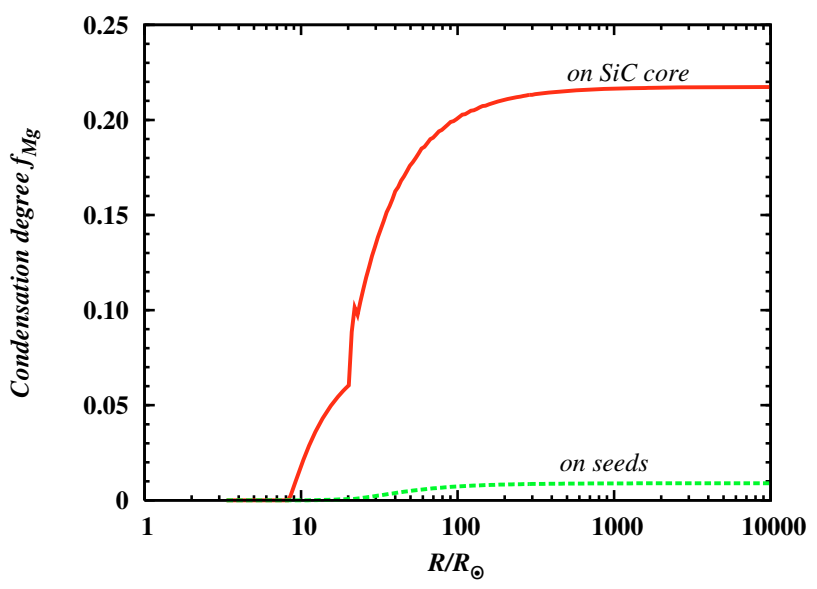

Fig. 5. Evolution of condensation degree of $\mathrm{Mg}$ with distance from the centre of the star for condensation of $\mathrm{MgS}$ (i) as mantle on top of a $\mathrm{SiC}$ core; and (ii) on seed particles (solid and dashed line, respectively).

onset of $\mathrm{MgS}$ formation the $\mathrm{SiC}$ core radius reaches $\propto 0.07 \mu \mathrm{m}$ as shown in Fig. 4. The results of MgS condensation on tiny seed nuclei are shown in Fig. 4 for comparison. The SiC core is big enough to allow the formation of a considerable thickness of the $\mathrm{MgS}$ coating, of order $0.02 \mu \mathrm{m}$, which results in a final grain size of $0.9 \mu \mathrm{m}$. This value is very close to the grain sizes required to explain the shape of the extinction feature in the spectra of carbon stars. We assume in the calculation that $\mathrm{SiC}$ grains do not grow anymore as soon as the formation of the $\mathrm{MgS}$ mantle begins. Figure 4 shows that the growth of $\mathrm{SiC}$ grains with and without termination due to $\mathrm{MgS}$ mantle formation does not change the grain size very much. Thus, a model with MgS mantle formation on SiC does not change noticeably the fraction of $\mathrm{Si}$ condensed in $\mathrm{SiC}$ dust, but results in a much higher value of the degree of condensation of $\mathrm{Mg}$ in $\mathrm{MgS}$ as compared to the case of growth on seeds. This is illustrated in Fig. 5.

A similar test calculation for $\mathrm{MgS}$ formation as a coating on carbon grains also gave sufficient thicknesses of $\mathrm{MgS}$ layers. Basically, condensation of $\mathrm{MgS}$ on carbon cores cannot be excluded, but from a physical point of view, $\mathrm{MgS}$ mantle formation is more likely on a $\mathrm{SiC}$ core, since the bond lengths and bonding properties of $\mathrm{SiC}$ are similar to that of $\mathrm{MgS}$. Laboratory experiments would be necessary to arrive at a final conclusion about the carriers of $\mathrm{MgS}$ mantles.

\section{Optical properties of grains with a SiC core and MgS mantle}

In order to study the difference in the optical properties of grains with coatings of $\mathrm{MgS}$ either on a $\mathrm{SiC}$ core or on a carbon core we calculate the absorption efficiency $C_{\lambda}^{\text {abs }}$ (i.e. the ratio of absorption to geometrical cross-section) in the small particle limit (cf. Bohren \& Huffman 1983)

$C_{\lambda}^{\mathrm{abs}}=4 x \operatorname{Im} \frac{\alpha}{4 \pi\left(r_{\mathrm{c}}+r_{\mathrm{m}}\right)^{3}}$

with

$$
\begin{aligned}
\alpha= & 4 \pi\left(r_{\mathrm{c}}+r_{\mathrm{m}}\right)^{3} \\
& \times \frac{\left(r_{\mathrm{c}}+r_{\mathrm{m}}\right)^{3}\left(\epsilon_{2}-1\right)\left(\epsilon_{1}+2 \epsilon_{2}\right)+r_{\mathrm{c}}^{3}\left(2 \epsilon_{2}+1\right)\left(\epsilon_{1}-\epsilon_{2}\right)}{\left(r_{\mathrm{c}}+r_{\mathrm{m}}\right)^{3}\left(\epsilon_{2}+2\right)\left(\epsilon_{1}+2 \epsilon_{2}\right)+r_{\mathrm{c}}^{3}\left(2 \epsilon_{2}-2\right)\left(\epsilon_{1}-\epsilon_{2}\right)} .
\end{aligned}
$$

Here $r_{\mathrm{c}}$ is the core radius, $r_{\mathrm{m}}$ the thickness of the mantle and $\epsilon_{1}$ and $\epsilon_{2}$ are the complex dielectric functions of the core and mantle material, respectively. $x$ is the size parameter $2 \pi\left(r_{\mathrm{c}}+r_{\mathrm{m}}\right) / \lambda$.

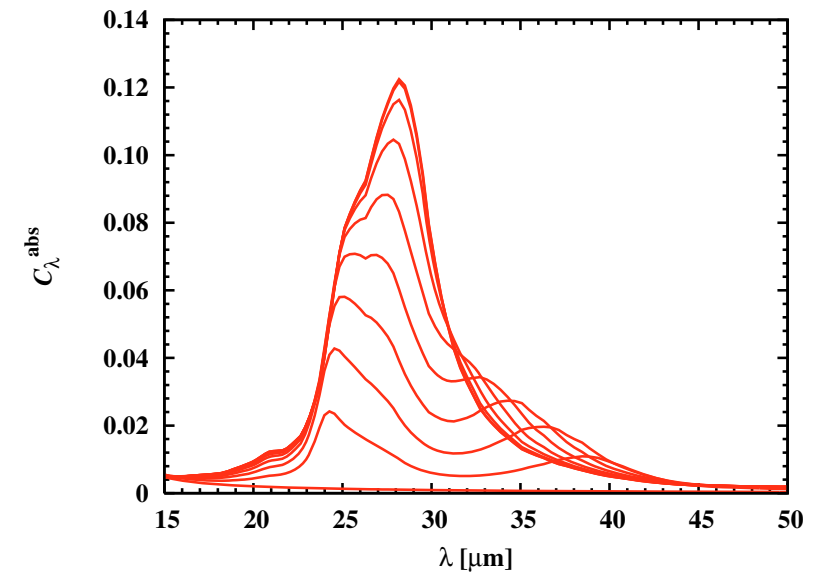

Fig. 6. Wavelength variation of the absorption efficiency $C_{\lambda}^{\text {abs }}$ of spherical grains with $\mathrm{SiC}$ core of radius $r_{\mathrm{c}}$ coated with $\mathrm{MgS}$ mantles of thickness $r_{\mathrm{m}}$ for a total grain radius $r_{\mathrm{c}}+r_{\mathrm{m}}$ of $0.1 \mu \mathrm{m}$ and for MgS-mantle thickness to total radius ratios from 0 to 1 in steps of 0.1 (from bottom to top).

The small particle approximation $x \ll 1$ is valid in our case since we are interested in wavelengths from the region from 10 to $40 \mu \mathrm{m}$ that are much larger than the size of the circumstellar dust particles.

Optical constants for MgS are taken from Begemann et al. (1994) for $\mathrm{Mg}_{x} \mathrm{Fe}_{1-x} \mathrm{~S}$ with $x=0.9$. For carbon we use data for the $\mathrm{BE}$ carbon dust of the evaluation of the data of Colangeli et al. (1993) by Zubko et al. (1996). For SiC we used the data for $\mathrm{SiC}$ from Laor \& Draine (1993). Extinction efficiencies have been calculated for $\mathrm{SiC}$ grains coated with $\mathrm{MgS}$ mantles and, for comparison, also for carbon grains with $\mathrm{MgS}$ mantles.

Figure 6 shows results for grains of total radius $r_{\mathrm{c}}+r_{\mathrm{m}}$ of $0.1 \mu \mathrm{m}$ and mantle thicknesses between 0 and $0.1 \mu \mathrm{m}$, i.e., for grains ranging in composition from pure $\mathrm{SiC}$ to pure $\mathrm{MgS}$ grains. The results show the rather broad $\mathrm{MgS}$ absorption band centred at about $26 \mu \mathrm{m}$, which in itself shows some structure. Additionally a secondary peak appears to be centred around $\approx 33 \ldots 38 \mu \mathrm{m}$ for moderate mantle thicknesses. Its position depends on the size ratio of core and mantle. This feature is not present in the extinction efficiencies of pure $\mathrm{MgS}$ or $\mathrm{SiC}$ grains and results from the particular run of the complex dielectric functions of the core and the coating in this wavelength region ${ }^{4}$.

Figure 7 shows the variation of the extinction coefficient with varying core to mantle size ratios - both for $\mathrm{MgS}$ grains with $\mathrm{SiC}$ and with carbon cores - in a different representation and an extended wavelength region that also covers the $11 \mu \mathrm{m}$ feature of $\mathrm{SiC}$. The total grain radius $r_{\mathrm{c}}+r_{\mathrm{m}}$, again, is $0.1 \mu \mathrm{m}$ and the fraction $r_{\mathrm{m}} /\left(r_{\mathrm{c}}+r_{\mathrm{m}}\right)$ is varied between 0 and 1 . In the upper part of the picture one recognises how the $\mathrm{SiC}$ feature disappears and the MgS feature appears as the composition of the grain varies from pure $\mathrm{SiC}$ to pure $\mathrm{MgS}$. The extra feature peaking at about $33 \ldots 38 \mu \mathrm{m}$, depending on the mantle thickness, is clearly seen in coatings of moderate, but not too small fraction of the total size. For thick coatings the extra feature disappears together with the $11 \mu \mathrm{m}$ feature from the $\mathrm{SiC}$ core. The feature is not very distinct and will probably hardly be detectable in many cases.

The particular feature peaking at about $33 \ldots 38 \mu \mathrm{m}$ is missing in the extinction of carbon grains coated with $\mathrm{MgS}$, as can

\footnotetext{
${ }^{4}$ It has been checked if this results from a Fröhlich mode in coated particles at a frequency where the denominator in Eq. (24) vanishes, cf. the discussion of this effect in Bohren \& Huffman (1983), but this seems not to be the case.
} 

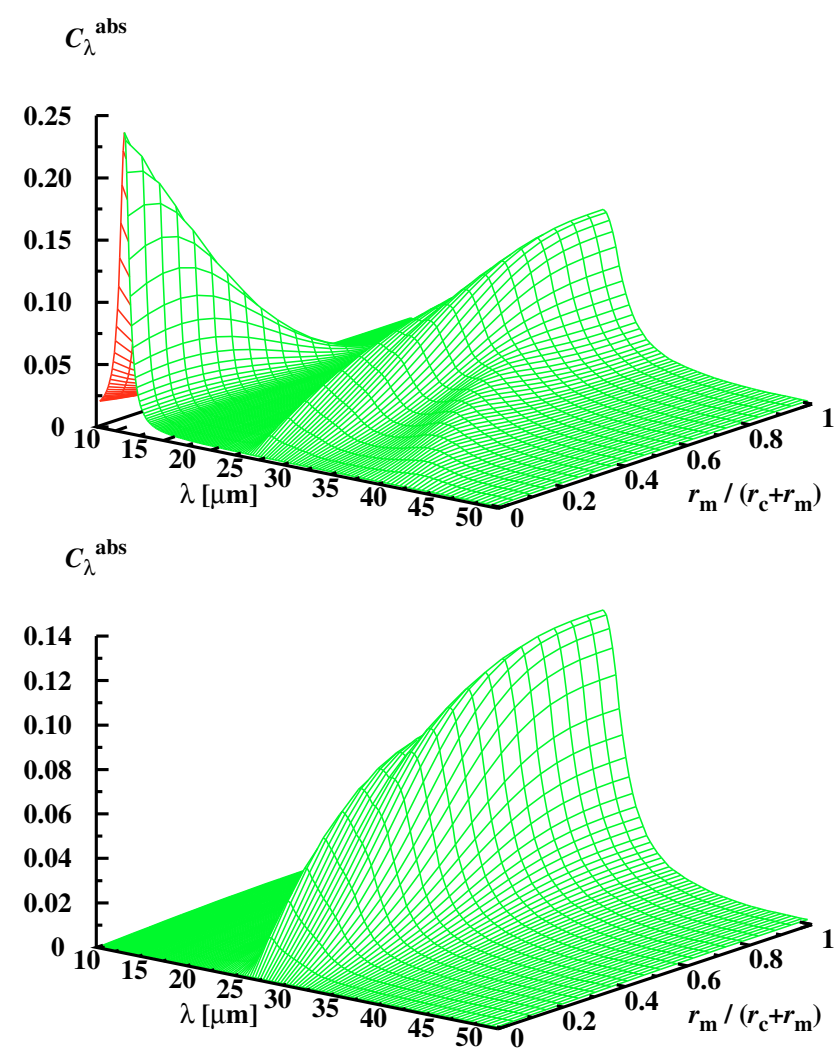

Fig. 7. Wavelength variation of the absorption efficiency $C_{\lambda}^{\text {abs }}$ of spherical grains with $\mathrm{SiC}$ (top) or carbon (bottom) core and $\mathrm{MgS}$ mantle with total radius of $0.1 \mu \mathrm{m}$. The $\mathrm{MgS}$ mantles range in thickness from zero to unity in steps of 0.05 for the ratio of mantle thickness $r_{\mathrm{m}}$ to total radius $r_{\mathrm{c}}+r_{\mathrm{m}}$, where $r_{\mathrm{m}}$ is the core radius. The extinction properties vary from that of pure $\mathrm{SiC}$ or carbon grains to that of pure $\mathrm{MgS}$ grains. An extra extinction feature centred on $33 \mu \mathrm{m}$ for $\mathrm{MgS}$ grains with $\mathrm{SiC}$ core shows up at moderate mantle thickness. This is missing in case of carbon cores.

be seen from the lower part of Fig. 7, because of the completely different properties of the dielectric function. Therefore this extra feature can be considered as an indicator of a silicon carbide core of $\mathrm{MgS}$ grains, if the presence of $\mathrm{MgS}$ is indicated by its absorption band around $26 \mu \mathrm{m}$. Observationally it was found by Volk et al. (2002) that the so called "30 $\mu$ m feature" of carbon stars shows in some cases some structure, and seems to consist of two overlapping features at $26 \mu \mathrm{m}$ and $33 \mu \mathrm{m}$. Our results for the extinction of $\mathrm{SiC}$-core-MgS-mantle grains indicate that one just sees that the $\mathrm{MgS}$ in outflows from carbon stars grows as mantles on silicon carbide cores. If it is not seen this probably does not mean that in this case $\mathrm{MgS}$ precipitates on carbon, but merely that the feature is not sufficiently well defined to be unambiguously detectable.

\section{Concluding remarks}

In this paper the mechanism of $\mathrm{MgS}$ formation in stellar outflows of AGB stars is studied. From some elementary considerations on the kinetics of $\mathrm{MgS}$ condensation we estimate a critical massloss rate of about $5 \times 10^{-4} M_{\odot} \mathrm{yr}^{-1}$ for efficient $\mathrm{MgS}$ formation if this would condense as a separate dust species via nucleation and subsequent growth. This value of the mass-loss rate is much higher than any observed mass-loss rate of AGB stars. Within the observed range of mass-loss rates of AGB stars, however, the amount of MgS that can condense on seed particles in stellar winds is much too low to explain the observed strong emission band from $\mathrm{MgS}$ in the infrared spectra of many carbon stars.

A model of heterogeneous growth of $\mathrm{MgS}$ on $\mathrm{SiC}$ precursor grains is proposed here as the most promising mechanism of $\mathrm{MgS}$ formation. Analysis of the equilibrium chemistry of sulphur compounds in a carbon rich mixture shows that the formation of $\mathrm{MgS}$ is strongly coupled to the process of silicon carbide condensation. If no $\mathrm{SiC}$ is formed in a stellar outflow, the sulphur is almost completely bound in SiS molecules. Only after significant consumption of the abundant gas phase $\mathrm{SiS}$ by $\mathrm{SiC}$ formation, is $\mathrm{H}_{2} \mathrm{~S}$ formed in abundance and reactions of this with $\mathrm{Mg}$ makes possible $\mathrm{MgS}$ condensation.

Our model calculations of $\mathrm{MgS}$ formation in stellar outflows show that only $\mathrm{MgS}$ growth as mantles atop SiC cores that condensed before the on-set of $\mathrm{MgS}$ formation, results in sufficiently high degrees of condensation of $\mathrm{Mg}$ into $\mathrm{MgS}$ (of the order of $\sim 0.2$ ) which are required so that $\mathrm{MgS}$ may be clearly visible in the infrared spectrum. Condensation of $\mathrm{MgS}$ on tiny seed particles would result in a fraction of the $\mathrm{Mg}$ condensed in $\mathrm{MgS}$ dust much too small to be detectable (of the order of only 0.01 ).

Additionally, we performed calculations of the extinction properties of grains with $\mathrm{SiC}$ core and $\mathrm{MgS}$ coating of various thicknesses. The presence of a $\mathrm{SiC}$ core inside of $\mathrm{MgS}$ grains results within some range of core/mantle volume ratios in a secondary peak near $33 \ldots 35 \mu \mathrm{m}$ in the broad emission band associated with $\mathrm{MgS}$, that seems to be observed in some spectra of AGB stars. This feature is absent if $\mathrm{MgS}$ forms as a mantle on carbon grains. We propose this feature as an indicator for the presence of a silicon carbide core of $\mathrm{MgS}$ grains, if the presence of $\mathrm{MgS}$ is indicated by its absorption band around $26 \mu \mathrm{m}$.

Acknowledgements. This work was supported by the Deutsche Forschungsgemeinschaft (DFG), Sonderforschungsbereich 439 "Galaxies in the Young Universe". S.Z. is supported in part also by the International Max-Planck Research School (IMPRS) Heidelberg.

\section{References}

Allende Prieto, C., Lambert, D. L., \& Asplund, M. 2001, ApJ, 556, L63 Allende Prieto, C., Lambert, D. L., \& Asplund, M. 2002, ApJ, 573, L137 Asplund, M., Grevesse, N., \& Sauval, A. J. 2005, in Cosmic Abundances as Records of Stellar Evolution and Nucleosynthesis, ed. T. G. Barnes III, \& F. N. Bash. (San Francisco: ASP), ASP Conf. Ser., 336, 25

Begemann, B., Dorschner, J., Henning, T., Mutschke, H., \& Thamm, E. 1994, ApJ, 423, L71

Bohren, C. F., \& Huffman, D. R. 1983, Absorption and Scattering of Light by Small Particles (New York: Wiley \& Sons)

Bowen, G. H. 1988, ApJ, 329, 299

Chaplin, W. J., Serenelli, A. M., Basu, S., et al. 2007, ApJ, 670, 872

Chase, M. W. 1998, NIST-JANAF Thermochemical Tables, 4th edn, Journal of Physical and Chemical Reference Data, Monograph, 9

Colangeli, L., Menella, V., Blanco, A., et al. 1993, ApJ, 418, 435

Cox, P. 1993, in Astronomical Infrared Spectroscopy, Future Observational Directions, ASP Conf. Ser., 41, 163

Daulton, T. L., Bernatowicz, T. J., Lewis, R. S., et al. 2003, Geochim. Cosmochim. Acta, 67, 4743

Ferrarotti, A. S., \& Gail, H.-P. 2006, A\&A, 447, 553

Feuchtinger, M. U., Dorfi, E. A., \& Höfner, S. 1995, A\&A, 273, 513

Fleischer, A. J., Gauger, A., \& Sedlmayr, E. 1991, A\&A, 242, L1

Fleischer, A. J., Gauger, A., \& Sedlmayr, E. 1992, A\&A, 266, 321

Forrest, W. J., Houck, J. R., \& McCarthy, J. F. 1981, ApJ, 248, 195

Gail, H.-P. 2003, In Astromineralogy, ed. Th. Hennig (Heidelberg: Springer), 55 Gail, H.-P., \& Sedlmayr, E. 1986, A\&A, 166, 225

Gail, H.-P., \& Sedlmayr, E. 1987, A\&A, 171, 197

Goebel, J. H., \& Moseley, S. H. 1985, ApJ, 290, L35 
Grevesse, N., \& Sauval, A. J. 1998, Space Sci. Rev., 85, 161

Grishko, V. I., Tereszchuk, K., Duley, W. W., \& Bernath, P. 2001, ApJ, 558, L129

Hashimoto, A. 1990, Nature, 347, 53

Henning, T., \& Stognienko, R. 1996, A\&A, 311, 291

Höfner, S., \& Dorfi, E. A. 1997, A\&A, 319, 648

Höfner, S., Jørgensen, U. G., Loidl, R., \& Aringer, B. 1998, A\&A, 340, 497

Hony, S., \& Bouwman, J. 2004, A\&A, 413, 981

Hony, S., Waters, L. B. F. M., \& Tielens, A. G. G. M. 2002, A\&A,390, 533

Hrivnak, B. J., Kwok, S., \& Kwok, S. 2000, ApJ, 535, 275

Jeong, K. S., Winters, J. M., Le Bertre, T., \& Sedlmayr, E. 2003, A\&A, 407, 191

Jiang, B. W., Szczerba, R., \& Deguchi, S. 1999, A\&A, 344, 918

Kimura, Y., Kurumada, M., Tamura, K., et al. 2005, A\&A, 442, 507

Laor, A., \& Draine, B. T. 1993, ApJ, 402, 441

Lattimer, J. M., Schramm, D. N., \& Grossman, L. 1978, ApJ, 219, 230

Lagadec, E., Zijlstra, A. A., Sloan, G. C., et al. 2007, MNRAS, 376, 1270

Leisenring, J. M., Markwick-Kemper, F., \& Sloan, G. C. 2008 [arXiv:0803.3067v1]

Michael, B. P., Nuth, J. A., III, \& Lilleleht, L. U. 2003, ApJ, 590, 579

Nuth, J. A., Moseley, H., Silverberg, R. F., Goebel, J. H., \& Moore, W. J. 1985, ApJ, 290, L41

Omont, A. 1993, in Astronomical Infrared Spectroscopy, Future Observational Directions, ASP Conf. Ser., 41, 87
Omont, A., Moseley, S. H., Cox, P., et al. 1995, ApJ, 454, 819

Papoular, R. 2000, A\&A, 362, L9

Pauling, L. 1960, The Nature of the Chemical Bond and the Structure of Molecules and Crystals, 3rd. edn. (Cornell University press)

Rouleau, F., \& Martin, P. G. 1991, ApJ, 377, 526

Sloan, G. C., Kraemer, K. E., Matsuura, M., et al. 2006, ApJ, 645, 1118

Szczerba, R., Henning, Th., Volk, K., \& Kwok, S. 1999, A\&A, 345, L39

van Loon, J. T., Groenewegen, M. A. T., de Koter, A., et al. 1999, A\&A, 351, 559

Vassiliadis, E., \& Wood, P. R. 1993, ApJ, 413, 641

Volk, K., Kwok, S., Herivnak, B. J., \& Szczerba, R. 2002, ApJ, 567, 412

Winters, J. M., Fleischer, A. J., Le Bertre, T., \& Sedlmayr, E. 1997, A\&A, 326, 305

Winters, J. M., Le Bertre, T., Jeong, K. S., Helling, C., \& Sedlmayr, E. 2000, A\&A, 361, 641

Yamamura, I., de Jong, T., Justtanont, K., Cami, J., \& Waters, L. B. F. M. 1998, Ap\&SS, 255, 351

Zubko, V. G., Menella, V., Colangeli, L., \& Busoletti, E. 1996, MNRAS, 282, 1321

Zijlstra, A. A., Matsuura, M., Wood, P. R., et al. 2006, MNRAS, 365, 370, 1961 